\title{
Testosterone/Estradiol Ratio in a Dog with Sertoli Cell Tumor-Seminoma Combination
}

\section{Doo-Won Song ${ }^{1}$ \\ Chang-Min Lee ${ }^{2}$ \\ Seung-Gon Kim ${ }^{3}$ \\ Min-Hee Kang ${ }^{1}$ \\ Dong-Wook Kim ${ }^{4}$ \\ Hwi-Yool Kim ${ }^{5}$ \\ Kidong Eom ${ }^{6}$ \\ Hee-Myung Park ${ }^{1, *}$}

'Department of Veterinary Internal Medicine, College of Veterinary Medicine, Konkuk University, Seoul 05029, Korea 2Department of Veterinary Internal Medicine, College of Veterinary Medicine and BK21 FOUR Program, Chonnam National University, Gwangju 61186, Korea

${ }^{3}$ Chungdam Animal Hospital, Seoul 06085 ,

Korea

${ }^{4}$ Cheonan Animal Medical Center,

Cheonan 31155, Korea

${ }^{5}$ Department of Veterinary Surgery,

College of Veterinary Medicine, Konkuk

University, Seoul 05029, Korea

${ }^{6}$ Department of Veterinary Radiology,

College of Veterinary Medicine, Konkuk

University, Seoul 05029, Korea

*Correspondence: parkhee@konkuk.ac.kr

\section{ORCID}

Doo-Won Song:

https://orcid.org/0000-0003-2596-9785

Chang-Min Lee:

https://orcid.org/0000-0002-7867-1053

Seung-Gon Kim:

https://orcid.org/0000-0002-2775-2822

Min-Hee Kang:

https://orcid.org/0000-0001-7304-5064

Dong-Wook Kim:

https://orcid.org/0000-0002-3978-6325 Hwi-Yool Kim:

https://orcid.org/0000-0001-6237-9958

Kidong Eom:

https://orcid.org/0000-0001-5032-5311

Hee-Myung Park:

https://orcid.org/0000-0002-4559-0417

Copyright $\odot$ The Korean Society of Veterinary Clinics
Abstract A 8-year-old male Golden Retriever dog with bilateral cryptorchidism presented for evaluation of symmetric alopecia and gynecomastia. Abdominal radiography and computed tomography revealed bilateral enlargement of the testicles in the abdominal cavity. The concentrations of estradiol and testosterone in the blood were evaluated, and the results revealed a low testosterone/estradiol ratio despite a normal concentration of estradiol. For correction, cryptorchid testicles were surgically removed. Grossly, the bilateral retroperitoneal cryptorchid testicles were enlarged, firm, and spherical. On histopathologic examination, the right abdominal cryptorchid testicle was diagnosed as a Sertoli cell tumor (SCT)-Seminoma (SEM) combination. The contralateral testicle in the abdomen was diagnosed as a Sertoli cell tumor. The clinical signs of feminization were improved after surgery. To our knowledge, this is the first report of a case of simultaneous SCT on one side and SCT-SEM combination on the other side with sex hormone profiles in a dog.

Key words canine, Sertoli cell tumor, seminoma, testosterone, estradiol. 


\section{Introduction}

Testicular tumors are common in both canine and human species, and they are the most common tumors to develop in cryptorchid testicles (1). Sertoli cell tumors (SCTs), seminomas (SEMs), and interstitial cell tumors (ICTs) are the most common types of testicular tumors $(1,10)$. Among testicular tumors, a combination of more than one tumor type in the same testicle occurs in $5.2 \%$ of cases $(3,10)$. Testicular tumors are approximately 13 times more likely to develop in dogs with cryptorchidism than in normal dogs $(1,3)$. Clinical signs of endocrine dysfunction caused by tumors have been commonly documented in SCTs and less often in ICTs and SEMs $(2,7,8,12)$. SCTs are estrogen-secreting tumors that cause clinical signs such as feminization with associated gynecomastia, skin hyperpigmentation, symmetric alopecia, and bone marrow suppression $(5,8,12)$. However, the shift in the balance between testosterone and estrogen may be a more important factor in the clinical signs than an absolute elevation of estrogen (8). This report describes a case of SCTSEM combined with feminization and alopecia in a dog, with measurement of the testosterone and estradiol ratio.

\section{Case Description}

An 8-year-old intact male Golden Retriever presented with generalized alopecia. On physical examination, generalized bilateral symmetric alopecia, enlargement of the nipples, and hyperpigmentation in the area of alopecia to macular melanosis were observed (Fig. 1A). There were no other remarkable findings on the dermatologic examination. A complete blood cell count revealed non-regenerative normocytic normochromic anemia. The serum biochemistry results were within normal ranges. C-reactive protein (CRP) levels were elevated (Table 1). Abdominal radiography and ultrasonography revealed retained bilateral testicles. Computed tomography confirmed bilateral testicular masses in the retroperitoneal cavity and prostatic enlargement (Fig. 2A). Sex hormones (testosterone and estradiol) were measured to identify the cause of alopecia, gynecomastia, and prostatic enlargement. Sex hormones were measured using a Cobas ${ }^{\circledR} 6000$ analyzer (Roche Diagnostics, Mannheim, Germany) and the normal range was referred to in a previous paper (8). Testosterone concentration $(0.26 \mathrm{ng} / \mathrm{mL})$ was lower than the reference $(1.95 \mathrm{ng} / \mathrm{mL}, 0.05-3.70 \mathrm{ng} / \mathrm{mL}$; median, minimum-maximum), but the estradiol concentration $(13.8 \mathrm{pg} / \mathrm{mL}$, ) was normal (18 pg/mL, 8.6-31.5 pg/mL). The testosterone/estradiol ratio (0.018) was also lower than normal $(9.6,0.58-35.8)$. The cryptorchid testicles were surgically removed. The gross appearance of the right and left cryptorchid testicles showed enlarged, firm, and spherical masses. The left and right testicles were $3.5 \mathrm{~cm}$ and $3 \mathrm{~cm}$ in diameter, respectively (Fig. 2B,
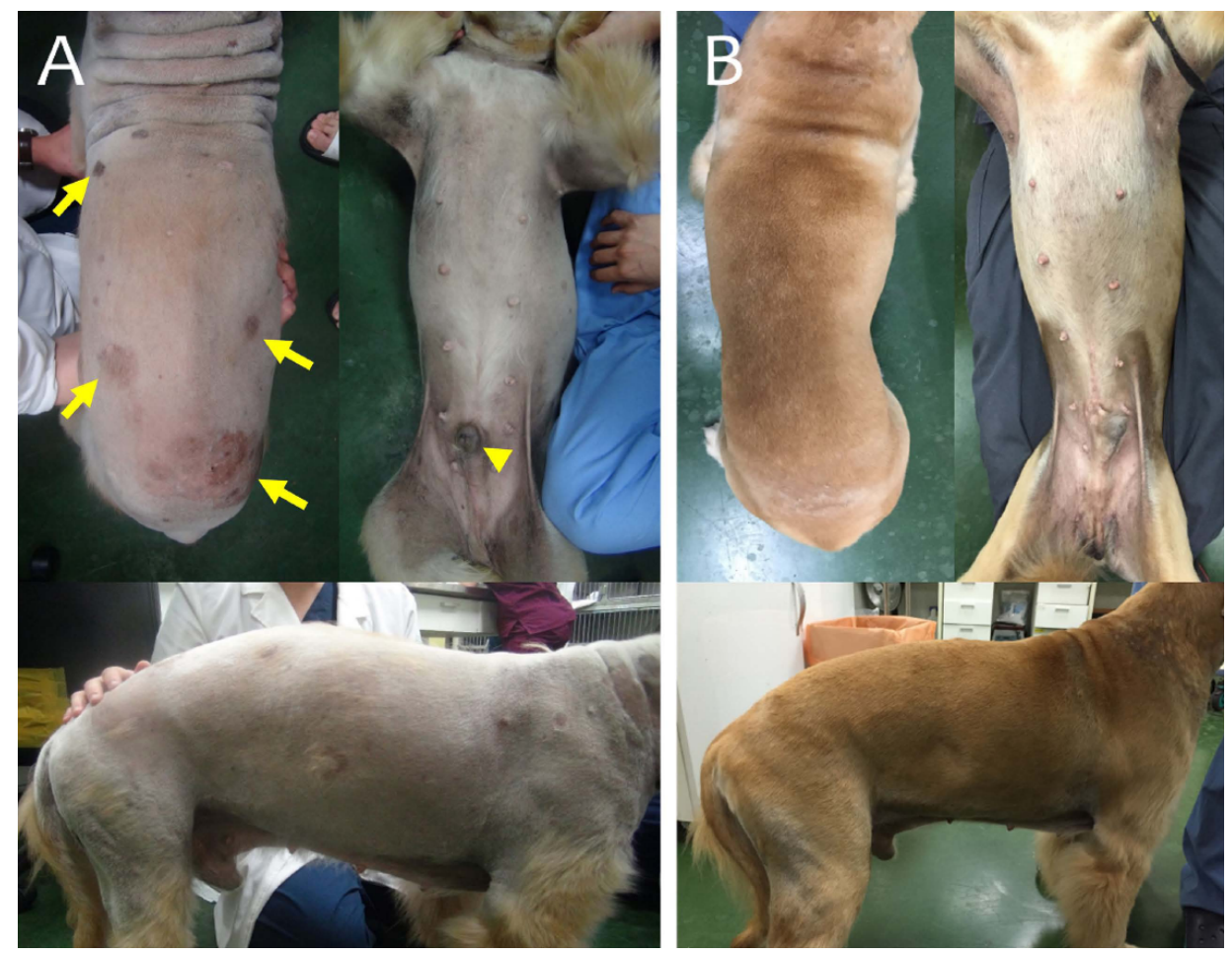

Fig. 1. (A) Clinical features that included symmetric alopecia. (B) After castration, a good response was observed within 2 months. Clinical signs of the skin such as hyperpigmentation (yellow arrow) and macular melanosis (yellow arrow head) gradually returned to normal. 
C). The cut surface of the right cryptorchid testicle revealed a firm, lobulated, and white to gray color with a homogeneous ivory color at the edge of the tumor. The left cryptorchid testicle was firm, lobulated, and white to gray in color. On histopathologic examination (IDEXX Laboratory, Westbrook,

Table 1. Complete blood count and serum biochemical tests in a dog with testicular tumors

\begin{tabular}{lccl}
\hline \multicolumn{1}{c}{ Parameters } & D0 & D14 & $\begin{array}{c}\text { Reference } \\
\text { interval }\end{array}$ \\
\hline WBC $\left(10^{3} / \mu \mathrm{L}\right)$ & 9.23 & 7.39 & $5.05-16.7$ \\
RBC $\left(10^{6} / \mu \mathrm{L}\right)$ & 4.89 & 5.55 & $5.65-8.87$ \\
HB $(\mathrm{g} / \mathrm{dL})$ & 11.1 & 12.5 & $13.1-20.5$ \\
HCT $(\%)$ & 31.2 & 35.7 & $37.3-61.7$ \\
Reticulocyte index & 0.7 & 1.2 & 1 \\
MCV & 63.7 & 64.3 & $61.6-73.5$ \\
MCH & 22.7 & 22.5 & $21.2-25.9$ \\
MCHC & 35.7 & 35 & $32-37.9$ \\
PLT $\left(10^{3} / \mu L\right)$ & 375 & 360 & $148-484$ \\
BUN $(\mathrm{mg} / \mathrm{dL})$ & 9 & 8 & $7-27$ \\
Creatinine $(\mathrm{mg} / \mathrm{dL})$ & 1.1 & 1.1 & $0.5-1.8$ \\
ALT $(\mathrm{U} / \mathrm{dL})$ & 14 & 17 & $10-100$ \\
AST $(\mathrm{U} / \mathrm{dL})$ & 22 & 35 & $0-50$ \\
ALP $(\mathrm{U} / \mathrm{dL})$ & 69 & 62 & $23-212$ \\
TP $(\mathrm{g} / \mathrm{dL})$ & 6.9 & 7.2 & $5.2-8.2$ \\
ALB $(\mathrm{g} / \mathrm{dL})$ & 3.0 & 3.2 & $2.3-4$ \\
CRP & 68 & 33 & $<10$ \\
\hline
\end{tabular}

$D$, days after operation; D0, before operation; WBC, white blood cells; RBC, red blood cells; Hb, hemoglobin; HCT, hematocrit; MCV, mean cell volume; $\mathrm{MCH}$, mean cell hemoglobin; $\mathrm{MCHC}$, mean cell hemoglobin concentration; PLT, platelet; BUN, blood urea nitrogen; ALT, alanine transaminase; AST, aspartate transaminase; ALP, alkaline phosphatase; TP, total protein; ALB, albumin; CRP, C-reactive protein.
Maine, USA), the left testicle, which showed a moderately well-demarcated and partially encapsulated neoplasm composed of atypical spindle and polygonal cells, was diagnosed as an SCT. The right testicle also showed the same histological findings as the left testicle, but the edges included rare nests of neoplastic cells containing moderate to abundant eosinophilic cytoplasm and central vesicular nucleus with 1-4 prominent magenta nucleoli. Therefore, the right testicle was diagnosed as a SCT-SEM combination. The clinical signs of the patient improved after orchidectomy (Fig. 1B). In addition, anemia and CRP levels improved after surgery (Table 1).

\section{Discussion}

The prevalence of testicular tumors varies from $11.2 \%$ to $27 \%$ in male dogs, with a higher incidence in the presence of cryptorchidism $(1,9)$. Although the incidence of tumors varies among studies, SEMs and ICTs occur frequently, and SCTs and mixed germ cell-sex cord-stromal cell tumors occur less frequently $(1,6,9)$. SCTs are the uncommon in dogs, but in dogs with cryptorchidism they are the most common tumors to develop (4). It is uncommon for more than one type of testicular tumor to develop in the same testicle. There have been previous reports of more than one type of testicular neoplasm occurs in 8.4\% (16/190), the most common combination of tumors SEM-ICT, followed by SEM-SCT and SCTICT (9). Our patient had tumors in both testicles, and one testis had developed more than one type of testicular tumor. SCT in the left testis and a combination of SCT and SEM in the right testis were diagnosed.

The patient also showed common clinical signs associated
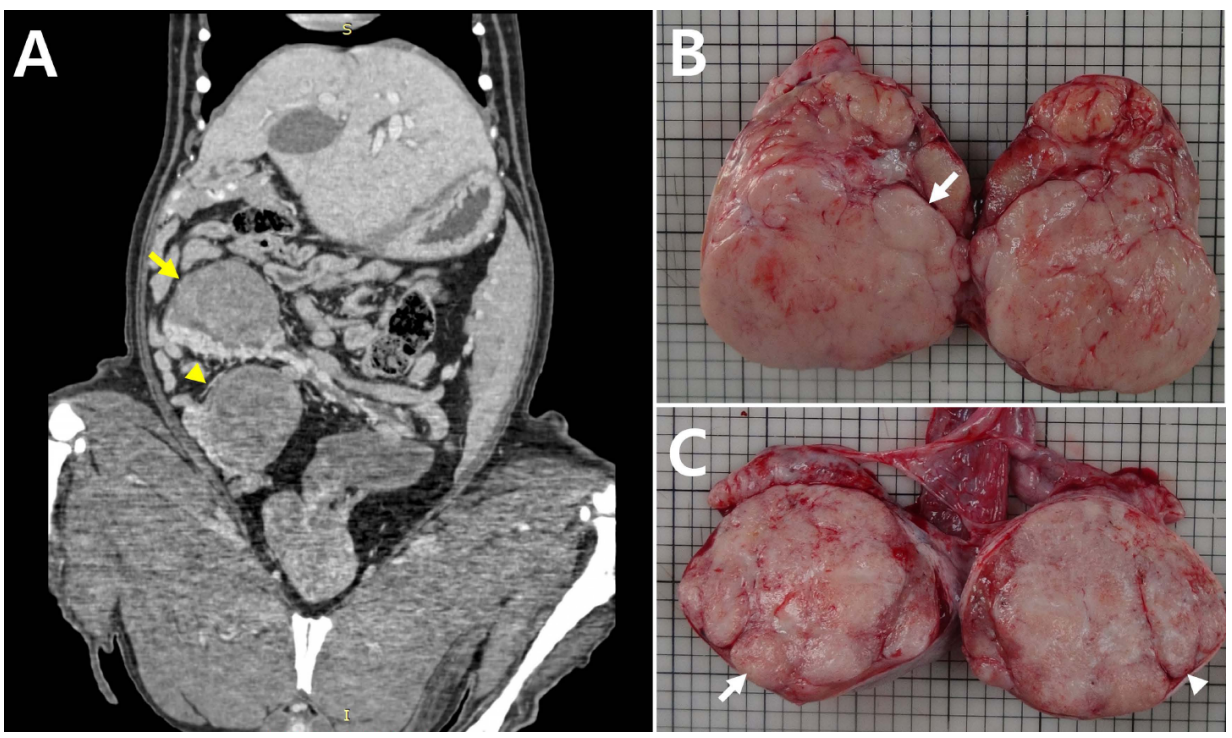

Fig. 2. (A) Computed tomographic image in a dog diagnosed as bilateral testicular tumors. Bilateral testicles were enlarged in the abdominal cavity: left testicle (yellow arrow, width $\times$ height $\times$ length, $\left.60 \times 68 \times 54 \mathrm{~mm}^{3}\right)$ and right testicle (yellow arrowhead, width $\times$ height $\times$ length, $64 \times 59 \times 59 \mathrm{~mm}^{3}$ ). (B, C) Gross appearance of bilateral cryptorchidism with testicular tumors after surgical removal. (B) The left testicle was diagnosed as a Sertoli cell tumor. Sectioned Sertoli cell tumor in the left testicle demonstrating its white-gray, lobulated appearance (white arrow). (C) The right testicle was diagnosed as a Sertoli cell tumor with seminoma. Sectioned right testicle demonstrating its white-gray, lobulated appearance (white arrow) with ivory, homogeneous appearance (white arrow head). 
with the SCT. These include symmetric alopecia, gynecomastia, and hyperpigmentation. Feminization and skin symptoms usually occur in SCTs and are associated with high estradiol concentrations (11); however, the dog had a normal estradiol level. Previous studies have shown that clinical symptoms are more related to the testosterone/estradiol ratio than estradiol alone, and clinical symptoms are associated with lower ratios (8). The study found that dogs with SCTs had higher levels of estradiol and lower levels of testosterone than normal dogs. In addition, estradiol levels were lower in dogs with SEM than in normal dogs. In this case, the effects of SCT and SEM were conflicting; therefore, the estradiol concentration appeared to be normal. However, as in other SCTs, the testosterone levels were low in this case. Testosterone levels may also be specific for SCTs. In this case, sex hormone profiles did not proceed after resection, but further studies are needed on the role of sex hormones in metastasis, recurrence, and complete resection of tumors.

In conclusion, this case report describes the sex hormone profiles related to the SEM-SCT combination. Our results suggest that the estradiol/testosterone ratio is more important than the estradiol concentration in the development of clinical symptoms. In addition, estradiol concentration may appear normal in the presence of the SCT-SEM combination. Therefore, it is necessary to measure the ratio of estradiol and testosterone together to avoid potential misunderstandings caused by the measurement of estriol alone. Sex hormone evaluation can differentiate the cause of clinical symptoms and the type of tumor, but is not sufficient for a definitive diagnosis. A comprehensive analysis is possible only with a combination of hormonal tests and histopathological evaluations.

\section{Acknowledgements}

The authors thank IDEXX Laboratories (Westbrook, Maine, USA) for the histopathology services.

\section{Conflicts of Interest}

The authors have no conflicting interests.

\section{References}

1. Grieco V, Riccardi E, Greppi GF, Teruzzi F, lermanò V, Finazzi M. Canine testicular tumours: a study on 232 dogs. J Comp Pathol 2008; 138: 86-89.

2. Grootenhuis AJ, van Sluijs FJ, Klaij IA, Steenbergen J, Timmerman MA, Bevers MM, et al. Inhibin, gonadotrophins and sex steroids in dogs with Sertoli cell tumours. J Endocrinol 1990; 127: 235242.

3. Hayes HM Jr., Pendergrass TW. Canine testicular tumors: epidemiologic features of 410 dogs. Int J Cancer 1976; 18: 482-487.

4. Hayes HM Jr, Wilson GP, Pendergrass TW, Cox VS. Canine cryptorchism and subsequent testicular neoplasia: case-control study with epidemiologic update. Teratology 1985; 32: 51-56.

5. Holst BS, Dreimanis U. Anti-Müllerian hormone: a potentially useful biomarker for the diagnosis of canine Sertoli cell tumours. BMC Vet Res 2015; 11: 166.

6. Liao AT, Chu PY, Yeh LS, Lin CT, Liu CH. A 12-year retrospective study of canine testicular tumors. J Vet Med Sci 2009; 71: 919 923.

7. Metzger FL Jr, Hattel AL, White DG. Hematuria, hyperestrogenemia, and hyperprogesteronemia due to a Sertoli-cell tumor in a bilaterally cryptorchid dog. Canine Pract 1993; 18: 32-35.

8. Mischke R, Meurer D, Hoppen HO, Ueberschär S, Hewicker-Trautwein M. Blood plasma concentrations of oestradiol-17beta, testosterone and testosterone/oestradiol ratio in dogs with neoplastic and degenerative testicular diseases. Res Vet Sci 2002; 73: 267272.

9. Nascimento HH, dos Santos A, Prante AL, Lamego EC, Tondo LA, Flores MM, et al. Testicular tumors in 190 dogs: clinical, macroscopic and histopathological aspects. Pesq Vet Bras 2020; 40: 525-535.

10. Nødtvedt A, Gamlem H, Gunnes G, Grotmol T, Indrebø A, Moe L. Breed differences in the proportional morbidity of testicular tumours and distribution of histopathologic types in a population-based canine cancer registry. Vet Comp Oncol 2011; 9: 4554.

11. Scott DW, Miller WH Jr, Griffin CE. Endocrine and metabolic diseases. In: Scott DW, Miller WH Jr., Griffin CE, editors. Muller \& Kirk's small animal dermatology. 5th ed. Philadelphia: Saunders. 1995: 682-686.

12. Suess RP Jr, Barr SC, Sacre BJ, French TW. Bone marrow hypoplasia in a feminized dog with an interstitial cell tumor. J Am Vet Med Assoc 1992; 200: 1346-1348. 